\title{
New modification Sentinel for Linear Parabolic Equation
}

\author{
LAIB Teldja ${ }^{1}$, Ayadi abdelhamid ${ }^{*}$ \\ 1,2 Department of Mathematics, \\ Laboratory of the dynamic systems and control University Larbi Ben M'Hidi, Oum El-Bouaghi-Algeria. \\ ${ }^{2}$ facmaths@yahoo.fr
}

\begin{abstract}
The purpose of this article is, firstly, to modify the old definition of the sentinel, introduced by J.L. Lions, so that we can separate the control support to the observation support and, secondly, to identify the pollution terms which arise in regular problems which are modeled by a linear parabolic equation. A new modification of the sentinel is used to identify pollution terms in the general case where the observation and the control supports are disjoint. The problem of finding a new modification sentinel is equivalent to finding the unique control of regular adjoint system of the parabolic equation that we solve.
\end{abstract}

Keywords: Regular Heat Equation; Controllability; Control optimal; Sentinel.

2010 MSC No: 35K05; 57R27

\section{Introduction}

We here use the new modification sentinel which change the classic definition of the sentinel method of J.-L. Lions (1992). The sentinel permits to distinguish between the pollution terms and the missing terms.

We are interested in the identification of the pollution terms which arise in regular parabolic problems. As it is well known, these problems generate distributed parameters which here are modeled by a heat linear parabolic equation. Here we want to estimate the pollution terms independently of the missing initial data ones. In general, the sentinel method is an important tool when one is interested in numerical simulations.

As we will see, the problem of finding the classical sentinel is equivalent to finding the optimal control of adjoint system of heat equation (see Ainseba B.-E. [1] and the general case in the book by J.-L. Lions [5]) where the control and the observation have their supports in the same open set. In our case, the new modification sentinel is used in its general setting since we consider two different open sets for the control and the observation.

Optimal control of distributed parameter systems governed by a system of parabolic equations is of special importance for propagation processing problems which are generally expressed by the resolution of the heat equation (see Fursikov [3, Brezis 2] ). The use of these equations may however leave a gap between the theoretical solutions and the experimental ones, then the use of optimal control allows to fill the gap, as it permits to optimize the distance between the two solutions.

As an immediate application, the existence of a new modification sentinel for a linear parabolic equation can be discussed, as we will see in the following section. We note that the backward problem appears under this form in the Lions sentinel theory [5].

\footnotetext{
${ }^{*}$ Corresponding author. Ayadi Abdelhamid

1 facmaths@yahoo.fr
} 
As noticed above, we will prove that the sentinel problem is equivalent to finding the unique optimal control of the adjoint system. We will follow the same techniques of the null-controllability problem for the heat equation which is well understood (see J. L. Lions 4, A. Omrane 8]). We state a more general null-controllability problem in this article.

The paper is organized as follows :

In section 2, we recall the Sentinel theory of Lions for linear heat problems with incomplete data.

In section 3, we use the null-controllability to prove the existence of the new sentinel.A well adapted version of the classical HUM method of Lions [4] is established.

In section 4, we show the existence of a non trivial new modification sentinel for the heat equation, where the control and observation have their supports in two different open sets.

\section{Towards a sentinel problem}

Let $\Omega$ be a bounded open subset of $\mathbb{R}^{d}, d \in \mathbb{N}^{*}$ with boundary $\Gamma$ of class $\mathcal{C}^{2}$. For $T>0$, we set $Q=(0, T) \times \Omega$, $\Sigma=(0, T) \times \Gamma$, and we consider the following linear heat problem in several dimensions:

$$
\frac{\partial y}{\partial t}-\Delta y=F \quad \text { in } Q
$$

with the following initial data of incomplete information :

$$
y(0)=\tau \hat{y}^{0} \quad \text { in } \Omega
$$

and with boundary condition

$$
y=0 \quad \text { on } \Sigma
$$

where $\tau \hat{y}^{0} \in L^{2}(\Omega)$ is unknown. (see Lions [5] page 156). Without loss of generality, we may assume that

$$
\left\|\hat{y}_{0}\right\|_{L^{2}} \leq 1 \quad \text { and } \tau \text { small } .
$$

Pollution terms appear in a part of the domain as follows:

$$
F=\lambda \hat{\xi}_{0} \quad \text { in } Q
$$

where $\lambda \hat{\xi}_{0} \in L^{2}(Q)$ is not known too.

Now we write the equation (1) in two equations:

$$
\begin{aligned}
& \mid \begin{array}{ccccc}
L y_{\lambda} & = & \hat{\xi}_{0} & \text { in } & Q \\
y_{\lambda} & = & 0 & \text { on } & \Sigma \\
y_{\lambda}(0) & = & 0 & &
\end{array} \\
& \mid \begin{array}{ccccc}
L y_{\tau} & = & 0 & \text { in } & Q \\
y_{\tau} & = & 0 & \text { on } & \Sigma \\
y_{\tau}(0) & = & y_{0} & &
\end{array}
\end{aligned}
$$

where $L$ and $L^{*}$ are given respectively by:

$$
\begin{gathered}
L=\frac{\partial}{\partial t}-\sum_{j=1}^{d} \frac{\partial^{2}}{\partial x_{j}^{2}} \\
L^{*}=-\frac{\partial}{\partial t}-\sum_{j=1}^{d} \frac{\partial^{2}}{\partial x_{j}^{2}}
\end{gathered}
$$


Then

$$
y=\tau y_{\tau}+\lambda y_{\lambda}
$$

And we have the following informations :

$$
y_{\tau}, \quad y_{\lambda} \text { in } L^{2}\left(0, T ; H_{0}^{1}(\Omega)\right)
$$

we have too

$$
t \rightarrow y_{\tau}, y_{\lambda}(t) \text { are continuous from }[0, T] \rightarrow L^{2}(\Omega)
$$

Let us recall that

$$
\text { | each of two systems (5) and (6) admits a unique solution }
$$

see Lions ([5])

The goal is to find a method to estimate the pollution term $\lambda \hat{\xi}_{0}$. Several methods can be used. The famous one is the least square method. However with this method, the pollution and initial unknown terms $\tau \hat{y}^{0}, \lambda \hat{\xi}_{0}$ are computed together and we cannot really separate them (see Lions [5], and Ainseba et al. [1] or [6] for the parabolic case).

Here, we use the new sentinel method which is a method of identifying one parameter, independently of the others. To have a chance to identify pollution, we observe the system in some open subset $\mathcal{O} \subset \Omega$ called observatory, during time $T$. We denote by $y_{o b s}$ this observation, which is known in $L^{2}(\mathcal{O} \times(0, T))$.

In the case of the heat equation, the observatory $\mathcal{O}$ can be chosen arbitrarily small.

We now will call back the notion of sentinel following the definition in the book chapter by Lions [5] (see also 7]). In this definition, the observation and the control may have different support sets but must be not disjoint.

Let $h_{0}$ be a given function on $(0, T) \times \mathcal{O}$ such that

$$
h_{0} \geq 0, \quad \int_{0}^{T} \int_{\mathcal{O}} h_{0} d x d t=1
$$

Let besides $\omega$ be an open and non empty subset of $\Omega$. For a control function $u \in L^{2}((0, T) \times \omega)$, we introduce the functional

$$
\mathcal{S}(\lambda, \tau)=\int_{0}^{T} \int_{\mathcal{O}} h_{0} y(t, x ; \lambda, \tau) d x d t+\int_{0}^{T} \int_{\omega} u y(t, x ; \lambda, \tau) d x d t
$$

We shall say that $S$ defines a sentinel (for the system (1)-(3) and (12) ) if there exists $u$ such that the pair $(u, S)$ satisfies to the following two conditions:

- $\mathrm{S}$ is insensitive at first order with respect to the missing terms $\tau \hat{y}^{0}$, which means

$$
\frac{\partial \mathcal{S}}{\partial \tau}(0,0)=0
$$

- and $u$ is of minimal norm in $L^{2}((0, T) \times \omega)$, in the sense

$$
\|u\|_{L^{2}((0, T) \times \omega)} \quad=\quad \inf _{v \in L^{2}((0, T) \times \omega)}\|v\| .
$$

In the above sentinel, one can not observe the state of the system somewhere in the domain, and control in another part of the domain $\Omega$. Our goal is to study this situation. 


\section{Controllability}

We give the function $G$ from $L^{2}(\omega)$ to $L^{2}(\mathcal{O})$ which verifies:

There is a positive constant c such that $\left\|G^{*} z\right\| \geq c\|z\|$, for all $z$ in $L^{2}(O)$

And, let be the regular parabolic system:

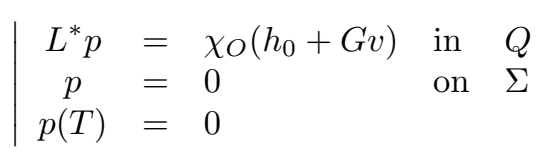

We divide this equation into two equations:

$$
\mid \begin{array}{clll}
L^{*} p^{0} & =\chi_{O} G v & \text { in } & Q \\
p^{0} & =0 & \text { on } & \Sigma \\
p^{0}(T) & =0
\end{array}
$$

and

$$
\mid \begin{array}{clll}
L^{*} p^{1} & =\chi_{O} h_{0} & \text { in } & Q \\
p^{1} & =0 & \text { on } & \Sigma \\
p^{1}(T) & = &
\end{array}
$$

where $\chi_{\omega}$ such that

$$
\chi_{\omega} u=\left\{\begin{array}{ccc}
u & \text { on } & (0, T) \times \omega \\
0 & \text { else }
\end{array}\right.
$$

For every given $v$ in $L^{2}(\omega)$, the system (16) admits a unique solution $p=p(v)$ in $L^{2}\left(0, T ; H_{0}^{1}(\Omega)\right.$ for the detail see [2]. then

The null controllability problem for 16 consists in finding $v$ in $L^{2}\left(0, T ; L^{2}(\omega)\right)$ such that if $p$ is solution of 16 .

$$
P(0)=0 .
$$

We can obtain this unique solution with the use of the following: First, we introduce the equation:

$$
\begin{array}{cccc}
L \varphi & = & 0 & \text { in } \\
\varphi & = & 0 & \text { on } \Sigma \\
\varphi(0) & = & \rho
\end{array}
$$

And we pose $v=-B^{*} \varphi$ and we define the operator $\Lambda$ by $\Lambda \rho=p^{0}(0)$ then we have

$$
\prec \Lambda \rho, \rho \succ=\left|B^{*} \varphi\right|_{L^{2}(\Omega)}^{2}
$$

Let $F$ be the completeness of $L^{2}(\Omega)$ with the norm $\|\rho\|_{F}^{2}=\left|B^{*} \varphi\right|_{L^{2}(\Omega)}^{2}$ and $F^{*}$ is the dual space of $F$, then the operator $\Lambda$ is ismorphism from $F$ to $F^{*}$ so for $-p^{1}(0) \in F^{*}$ there existe a $\rho \in F$ such that $p^{0}(0)+p^{1}(0)=0=p(0)$.

\section{Existence of a non trivial new modification sentinel}

Now, we can give the new definition of sentinel which one can observe somewhere in the domain, and control in another part of the domain $\Omega$

we introduce the functional

$$
\mathcal{S}(\lambda, \tau)=\int_{0}^{T} \int_{\mathcal{O}}\left(h_{0}+G(u)\right) y(t, x ; \lambda, \tau) d x d t .
$$

Where $y$ is the solution of the system 1

We shall say that $S$ defines a sentinel (for the system (1)-( 3 ) and (12)) if there exists $u$ such that the system 3 have unique solution and the pair $(u, S)$ satisfies to the following two conditions: 
- $\mathrm{S}$ is insensitive at first order with respect to the missing terms $\tau \hat{y}^{0}$, which means

$$
\frac{\partial \mathcal{S}}{\partial \tau}(0,0)=0
$$

- and $u$ is of minimal norm in $L^{2}((0, T) \times \omega)$, in the sense

$$
\|u\|_{L^{2}((0, T) \times \omega)}=\inf _{v \in L^{2}((0, T) \times \omega)}\|G(v)\| .
$$

The classical point of view of Lions lies on $h_{o}$ and $u$, having their supports in the same open set of observation $\mathcal{O}=\omega$. In this case, the question of the existence of a sentinel such that 23 holds is evident. Indeed, $h_{o}=-G(u)$ is a solution, and the only question is the calculus of the optimal control (24).

The point of view considered here is a sentinel notion defined by the function $h_{o}$, an observation $y_{\text {obs }}$ and a control $u$, but with $h_{o}$ having its support in $\mathcal{O}$ and $u$ of support in $\omega$ with $\omega \neq \mathcal{O}$. In this case, the existence of a sentinel is not guaranted.

\subsection{The identification of the pollution term}

We deduce that 23 is equivalent to

$$
\mathcal{S}(\lambda, \tau)=\int_{0}^{T} \int_{\mathcal{O}}\left(h_{0}+G(u)\right) y_{\tau}(t, x ; \lambda, \tau) d x d t=0,
$$

Now multiplying 16 by $y_{\tau}$ and integrating by parts we find:

$$
\int_{Q} y_{\tau} L^{*} p d x d t=\int_{Q} p y_{\tau} d x d t+\int_{\Omega} p(T) y_{\tau}(T) d x-\int_{\Omega} p(0) y_{\tau}(0) d x
$$

$+\int_{\Sigma} \frac{\partial y_{\tau}}{\partial \nu} p d \sigma-\int_{\Sigma} \frac{\partial p}{\partial \nu} y_{\tau} d \sigma$

$$
=\int_{0}^{T} \int_{\Omega}\left(h_{0}+G(u)\right) y_{\tau} d x d t
$$

because that

and

$$
\int_{\Omega} p(T) y_{\tau}(T) d x-\int_{\Omega} p(0) y_{\tau}(0) d x=0
$$

So, we deduce:

$$
\int_{\Sigma} \frac{\partial y_{\tau}}{\partial \nu} p d \sigma-\int_{\Sigma} \frac{\partial p}{\partial \nu} y_{\tau} d \sigma=0
$$

$$
\int_{0}^{T} \int_{\mathcal{O}} y_{\tau}\left(h_{0}+G(u) d x d t=\frac{\partial S}{\partial \tau}(0,0)=0\right.
$$

due to the fact that, $y_{\tau}$ is solution of 6 , and then

where

$$
S(\lambda, \tau)=S(0,0)+\tau \frac{\partial S}{\partial \tau}(0,0)+\lambda \frac{\partial S}{\partial \lambda}(0,0) \text { then } S_{o b s}=S(0,0)+\lambda \frac{\partial S}{\partial \lambda}(0,0)
$$

$$
S_{\text {obs }}=\int_{0}^{T} \int_{O}\left(h_{0}+G(u)\right) y_{o b s} d x d t \quad \text { and } \frac{\partial S}{\partial \lambda}(0,0)=\int_{0}^{T} \int_{\Omega} y_{\lambda}\left(h_{0}+G(u)\right) d x d t=\int_{Q} \hat{\xi}_{0} p d x d t
$$

Then

$\int_{Q}\left(\lambda \hat{\xi}_{0}\right) p d x d t=S_{o b s}-S(0,0)=S_{o b s}$, since $S(0,0)=0$

If $G$ is equal to identity then we have the classical definition of the sentinel 13 


\section{References}

[1] B.E. Ainseba, J.P. Kernevez R. Luce, Identification de paramètres dans des problèmes non linéaires à données incomplètes, M2AN Mathematical Modelling and Numerical Analysis, 28(3), (1994), 313-328.

[2] H. Brezis, Analyse fonctionnelle thorie et apllications, 2ê tirage, MASSON Paris New York Barcelone Milan Mexico Seo Paulo (1987)

[3] A. Fursikov, O. Yu. Imanuvilov, Controllability of evolution equations, Lecture Notes, Research Institute of Mathematics, Seoul National University, Korea (1996).

[4] J. L. Lions, Contrôlabilité exacte, stabilisation et perturbations des systèmes distribués, Vol. 1. Contrôlabilité exacte, Masson, Paris (1988). https://doi.org/10.1090/s0273-0979-1990-15909-9

[5] J. L. Lions, Sentinelles pour les systèmes distribués à données incomplètes, Masson, Paris (1992).

[6] Y. Miloudi, O. Nakoulima A. Omrane, A Method for Detecting Pollution in Dissipative Systems with Incomplete Data, ESAIM:Proceedings, 17(2007) 67-79. https://doi.org/10.1051/proc:071706

[7] Y. Miloudi,O. Nakoulima A. Omrane, On the instantaneous sentinels in pollution problems of incomplete data,Inverse Problems in Science and Engineering, 17(4), (2009), 451-459. https://doi.org/10.1080/17415970802015948

[8] A. Omrane, Some Aspects of the Sentinel Method for Pollution Problems, Air Quality - Monitoring and Modeling, ISBN 978-953-51-0161-1, InTech, Chap 9, (2012) 185-205. 